University of Wollongong

Research Online

Australian Institute for Innovative Materials -

Papers

Australian Institute for Innovative Materials

$1-1-2017$

Capillary-Induced Ge Uniformly Distributed in N-Doped Carbon Nanotubes with Enhanced Li-Storage Performance

Haipeng Guo

University of Wollongong, hg476@uowmail.edu.au

Boyang Ruan

University of Wollongong, br970@uowmail.edu.au

Lili Liu

University of Wollongong, II422@uowmail.edu.au

Lei Zhang

University of Wollongong, Iz755@uowmail.edu.au

Zhanliang Tao

University of Wollongong, Nankai University, ztao@uow.edu.au

See next page for additional authors

Follow this and additional works at: https://ro.uow.edu.au/aiimpapers

Part of the Engineering Commons, and the Physical Sciences and Mathematics Commons

Research Online is the open access institutional repository for the University of Wollongong. For further information contact the UOW Library: research-pubs@uow.edu.au 


\title{
Capillary-Induced Ge Uniformly Distributed in N-Doped Carbon Nanotubes with Enhanced Li-Storage Performance
}

\author{
Abstract \\ Germanium (Ge) is a prospective anode material for lithium-ion batteries, as it possesses large theoretical \\ capacity, outstanding lithium-ion diffusivity, and excellent electrical conductivity. Ge suffers from drastic \\ capacity decay and poor rate performance, however, owing to its low electrical conductivity and huge \\ volume expansion during cycling processes. Herein, a novel strategy has been developed to synthesize a \\ $\mathrm{Ge}$ at N-doped carbon nanotubes (Ge at N-CNTs) composite with Ge nanoparticles uniformly distributed \\ in the N-CNTs by using capillary action. This unique structure could effectively buffer large volume \\ expansion. When evaluated as an anode material, the Ge at N-CNTs demonstrate enhanced cycling \\ stability and excellent rate capabilities.

\section{Keywords} \\ enhanced, nanotubes, carbon, n-doped, distributed, uniformly, ge, performance, li-storage, capillary- \\ induced \\ Disciplines \\ Engineering | Physical Sciences and Mathematics

\section{Publication Details} \\ Guo, H., Ruan, B., Liu, L., Zhang, L., Tao, Z., Chou, S., Wang, J. \& Liu, H. (2017). Capillary-Induced Ge \\ Uniformly Distributed in N-Doped Carbon Nanotubes with Enhanced Li-Storage Performance. Small, 13 \\ (28), 1700920-1-1700920-7.
}

\section{Authors}

Haipeng Guo, Boyang Ruan, Lili Liu, Lei Zhang, Zhanliang Tao, Shulei Chou, Jiazhao Wang, and Hua-Kun Liu 


\title{
Capillary-Induced Ge Uniformly Distributed in $\mathrm{N}$-Doped Carbon Nanotubes with Enhanced Li-Storage Performance
}

\author{
Haipeng Guo, Boyang Ruan, Lili Liu, Lei Zhang, Zhanliang Tao, Shulei Chou, \\ Jiazhao Wang, * and Huakun Liu*
}

Germanium (Ge) is a prospective anode material for lithium-ion batteries, as it possesses large theoretical capacity, outstanding lithium-ion diffusivity, and excellent electrical conductivity. Ge suffers from drastic capacity decay and poor rate performance, however, owing to its low electrical conductivity and huge volume expansion during cycling processes. Herein, a novel strategy has been developed to synthesize a Ge@N-doped carbon nanotubes (Ge@N-CNTs) composite with Ge nanoparticles uniformly distributed in the $N$-CNTs by using capillary action. This unique structure could effectively buffer large volume expansion. When evaluated as an anode material, the Ge@N-CNTs demonstrate enhanced cycling stability and excellent rate capabilities.

\begin{abstract}
Lithium-ion batteries (LIBs), as the most advanced energy storage device, have been widely applied in electrical vehicles, portable electronics, and smart grids. ${ }^{[1,2]}$ The commercialized graphite anodes, however, cannot satisfy the demand for high-energy, next-generation batteries because of the low theoretical specific capacity $\left(372 \mathrm{mAh} \mathrm{g}^{-1}\right)$ of graphite. ${ }^{[3]}$ Therefore, anode materials with high capacity have been introduced, such as $\mathrm{Si}, \mathrm{Ge}$, and Sn. ${ }^{[4,5]}$ Among these, germanium has attracted considerable attention as a superior anode material owing to its excellent electrical conductivity, outstanding lithium-ion diffusivity, and large theoretical capacity. ${ }^{[6-9]}$ Similar to other anode materials (like silicon and
\end{abstract}

H. Guo, B. Ruan, L. Liu, L. Zhang, Prof. Z. Tao, Dr. S. Chou, Prof. J. Wang, Prof. H. Liu

Institute for Superconducting and Electronic Materials University of Wollongong

Squires Way, North Wollongong, NSW 2500, Australia

E-mail: jiazhao@uow.edu.au; hua@uow.edu.au

Prof. Z. Tao

Key Laboratory of Advanced Energy Materials

Chemistry (Ministry of Education)

College of Chemistry

Nankai University

Tianjin 300071, China

DOI: $10.1002 / \mathrm{smll} .201700920$ tin), however, Ge also suffers from drastic capacity decay and poor rate performance owing to the severe volume changes (about 300\%) during repeated charge/discharge processes. ${ }^{[10]}$ One approach to improving the electrochemical performance is to use nanosized materials, especially $1 \mathrm{D}$ nanotubes or nanowire structures, due to their short lithium-ion diffusion lengths and excellent electrical conductivities. ${ }^{[11-17]}$ Many strategies have been developed to synthesize 1D structured materials, such as the hydrothermal, ${ }^{[15,16]}$ chemical-vapor deposition, ${ }^{[18-22]}$ electrospinning, ${ }^{[23]}$ and chemical polymerization ${ }^{[24]}$ methods. Another method is to use a carbon matrix, which could accommodate volume changes, enhance electrical conductivity of the electrode, and serve as an active material for lithium storage. ${ }^{[25-28]}$ In particular, nanoparticles encapsulated in carbon nanotubes represent the most desirable structure, which could not only benefit from the advantages of $1 \mathrm{D}$ structure, but also provide void spaces to buffer the volume changes of active materials. ${ }^{[29-32]}$ For example, Chu and co-workers prepared peapod-like $\mathrm{Ge} / \mathrm{CN}_{x}$ with $\mathrm{Ge}$ incorporated in a $\mathrm{CN}_{x}$ layers by using a chemical polymerization method to coat a polypyrrole layer on the surfaces of $\mathrm{GeO}_{2}$ nanowires. ${ }^{[29]} \mathrm{Yu}$ and co-workers synthesized carbonnanofiber-encapsulated $\mathrm{Ge}$ nanoparticles through the electrospinning method. ${ }^{[32]}$ The active material nanoparticles were not very uniformly distributed within the carbon nanotubes, however, which may mean that the void spaces cannot 


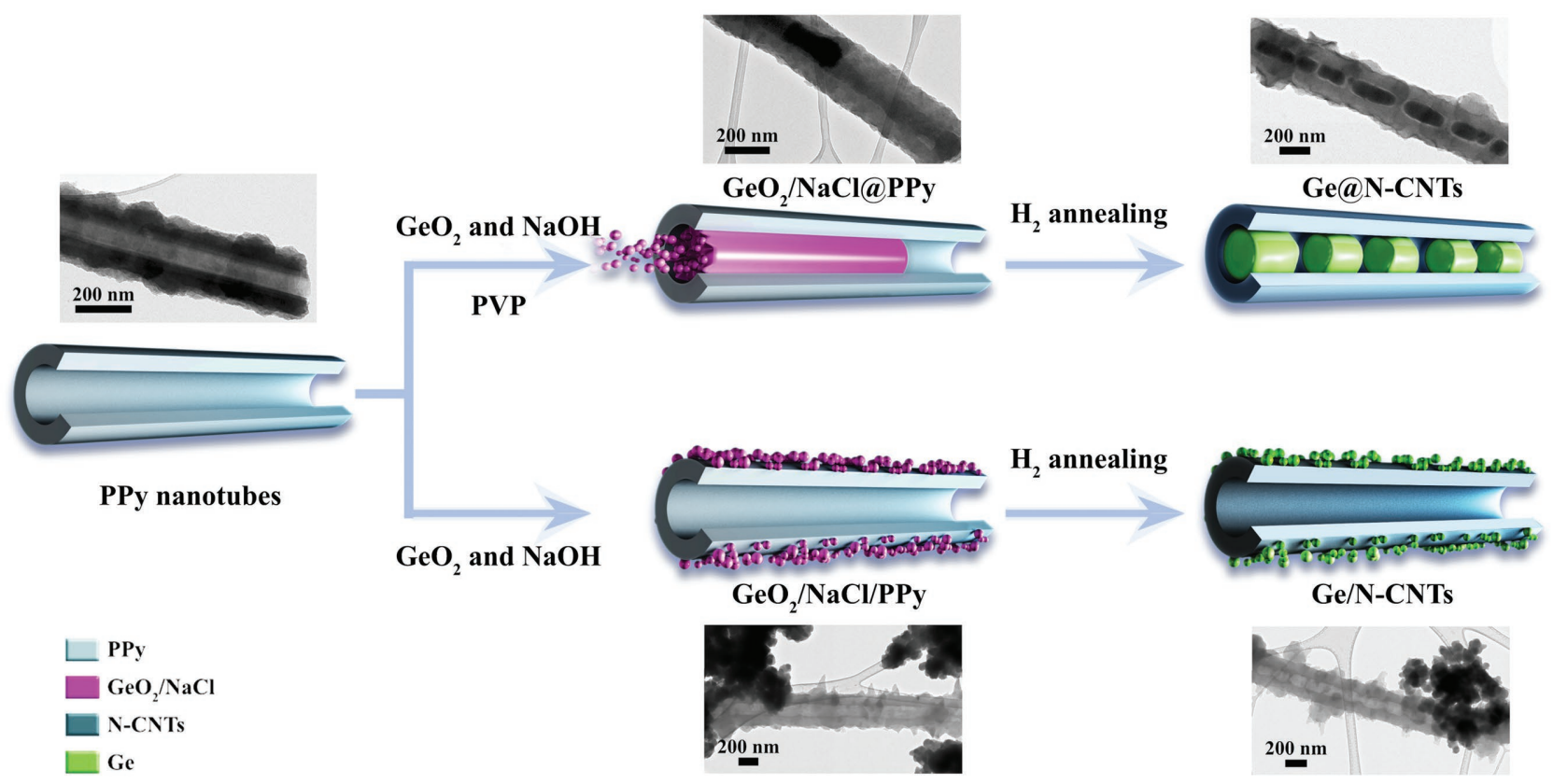

Figure 1. Illustration of the synthesis processes for the Ge@N-CNTs and Ge/N-CNTs composites.

be fully utilized to accommodate volume changes. Therefore, it is extremely attractive to design a novel strategy to synthesize 1D structured materials with uniformly distributed active materials.

Capillary action is a facile strategy to synthesize novel 1D structured materials through filling the hollow cavities of nanotubes with the chosen materials. ${ }^{[33]}$ Numerous efforts have been devoted to drawing different foreign materials in the liquid or molten phase into the hollow inner cavities by using capillary action to synthesize 1D structured materials. ${ }^{[34-39]}$ The surface tension of the foreign materials, however, is the most important parameter for successful filling, which should be less than $200 \mathrm{mN} \mathrm{m}^{-1} \cdot{ }^{[40]}$ Hence, only materials with low surface tension could be introduced into the hollow cavities by using capillary action. Therefore, it is still a big challenge to introduce high surface tension materials into the inner hollow cavities to fabricate 1D structured materials, which are expected to provide breathtaking opportunities for practical applications and fundamental research.

Herein, we present a feasible strategy to synthesize coreshell $\mathrm{GeO}_{2} / \mathrm{NaCl} @$ polypyrrole nanotubes by utilizing capillary action, which could be further transformed into a 1D Ge@Ndoped carbon nanotubes (Ge@N-CNTs) composite, with Ge nanoparticles uniformly distributed in the N-CNTs. The successful fabrication of Ge nanoparticles uniformly encapsulated in N-CNTs lies in two key aspects. First, as a high surface tension material, $\mathrm{GeO}_{2}\left(250 \mathrm{mN} \mathrm{m}^{-1}\right)$ could not be directly introduced into the hollow cavities in the molten phase by using capillary action. ${ }^{[41]}$ Even after it is dissolved in $\mathrm{NaOH}$ solution to form a liquid phase, the surface tension of the solution is still larger than the cut-off value, which also cannot be introduced into the hollow cavities by using capillary action. The surface tension could be decreased, however, through adding poly(vinylpyrrolidone) (PVP), and then the solution could be induced into the robust PPy nanotubes to form the core-shell
$\mathrm{GeO}_{2} / \mathrm{NaCl} @$ polypyrrole (PPy) nanotubes composite by utilizing capillary action. Second, through reducing $\mathrm{GeO}_{2}$ to $\mathrm{Ge}$ and removing $\mathrm{NaCl}$ with a further annealing and centrifuging treatment, a composite of Ge nanoparticles uniformly distributed in the N-CNTs could be obtained. The uniformly distributed Ge nanoparticles could effectively utilize the void spaces provided by the unique 1D structure during cycling, which could preserve the original structure of the Ge@N-CNTs and achieve prolonged cycling stability. When investigated its lithium storage performance, the Ge@N-CNTs demonstrated enhanced cycling stability and excellent rate capacity.

The synthesis process to fabricate the Ge@N-CNTs and Ge/N-CNTs composites is illustrated in Figure 1. First, uniform PPy nanotubes with a hollow inner cavity and open tips, which are favorable for capillary action, are synthesized through the polymerization method in a relatively large quantity. Second, the PPy nanotubes are mixed with a $\mathrm{GeO}_{2}$ and $\mathrm{NaOH}$ aqueous solution, followed by adjusting the $\mathrm{pH}$ of the solution to 7 with diluted $\mathrm{HCl}$. Then, PVP is added into the solution, which can decrease the surface tension and thus increase the effect of capillary action to fill the inner hollow cavities with $\mathrm{GeO}_{2} / \mathrm{NaCl}$ in an aqueous solution. ${ }^{[42]}$ Therefore, by utilizing capillary action, a core-shell $\mathrm{GeO}_{2} /$ $\mathrm{NaCl} @ P P y$ nanotubes composite is formed through inducing $\mathrm{GeO}_{2} / \mathrm{NaCl}$ into the inner hollow cavities after drying at $60{ }^{\circ} \mathrm{C}$. Finally, the unique 1D structured Ge@N-CNTs composite with $\mathrm{Ge}$ nanoparticles uniformly encapsulated in $\mathrm{N}-\mathrm{CNTs}$ is obtained through a carbonization and annealing process in $\mathrm{H}_{2}$ atmosphere, followed by the removal of $\mathrm{NaCl}$ by centrifuging. As shown in the transmission electron microscope (TEM) images (Figure 1) and the scanning electron microscope (SEM) images (Figure S1, Supporting Information), the PPy nanotubes with an inner cavity diameter of about $100 \mathrm{~nm}$ and open tips can be directly used for capillary action without any further treatment. The successful synthesis 
of core-shell $\mathrm{GeO}_{2} / \mathrm{NaCl} @$ PPy nanotubes could be further confirmed by elemental mapping images (Figure 2), which reveal that $\mathrm{GeO}_{2}$ and $\mathrm{NaCl}$ are completely filled into the hollow inner cavity and uniformly distributed along the axial direction of a nanotube. The $\mathrm{GeO}_{2} / \mathrm{NaCl}$ could not be induced into the hollow cavities of PPy nanotubes, however, without adding PVP. This was because the surface tension of $\mathrm{GeO}_{2} / \mathrm{NaCl}$ solution is too high to draw $\mathrm{GeO}_{2} / \mathrm{NaCl}$ into the inner hollow cavities. All of the $\mathrm{GeO}_{2} / \mathrm{NaCl}$ nanoparticles are dispersed on the outside of the PPy nanotubes instead of filling the hollow cavities. Therefore, Ge/N-CNTs with Ge nanoparticles aggregated on the outside of the N-CNTs could be obtained after further annealing and centrifugal treatment (Figure 1 and Figure S2, Supporting Information). This implies that the PVP could increase the effect of capillary action through decreasing the surface tension. Moreover, the distribution of the nanoparticles in the composite could be easily tuned by adjusting the surface tension. During the heating process, the N-CNTs content was totally burned out, and the $\mathrm{Ge}$ was oxidized into $\mathrm{GeO}_{2}$ with increasing temperature. Therefore, based on the weight of $\mathrm{GeO}_{2}$, the content of $\mathrm{Ge}$ can be calculated. By using this method, the Ge contents are determined to be 70.5 and $72.5 \mathrm{wt} \%$ in the Ge/N-CNTs and Ge@N-CNTs, respectively, according to the thermogravimetric analysis data (Figure S3, Supporting Information).

The X-ray diffraction (XRD) patterns are manifested in Figure 3a. The XRD peaks of the Ge@N-CNTs and
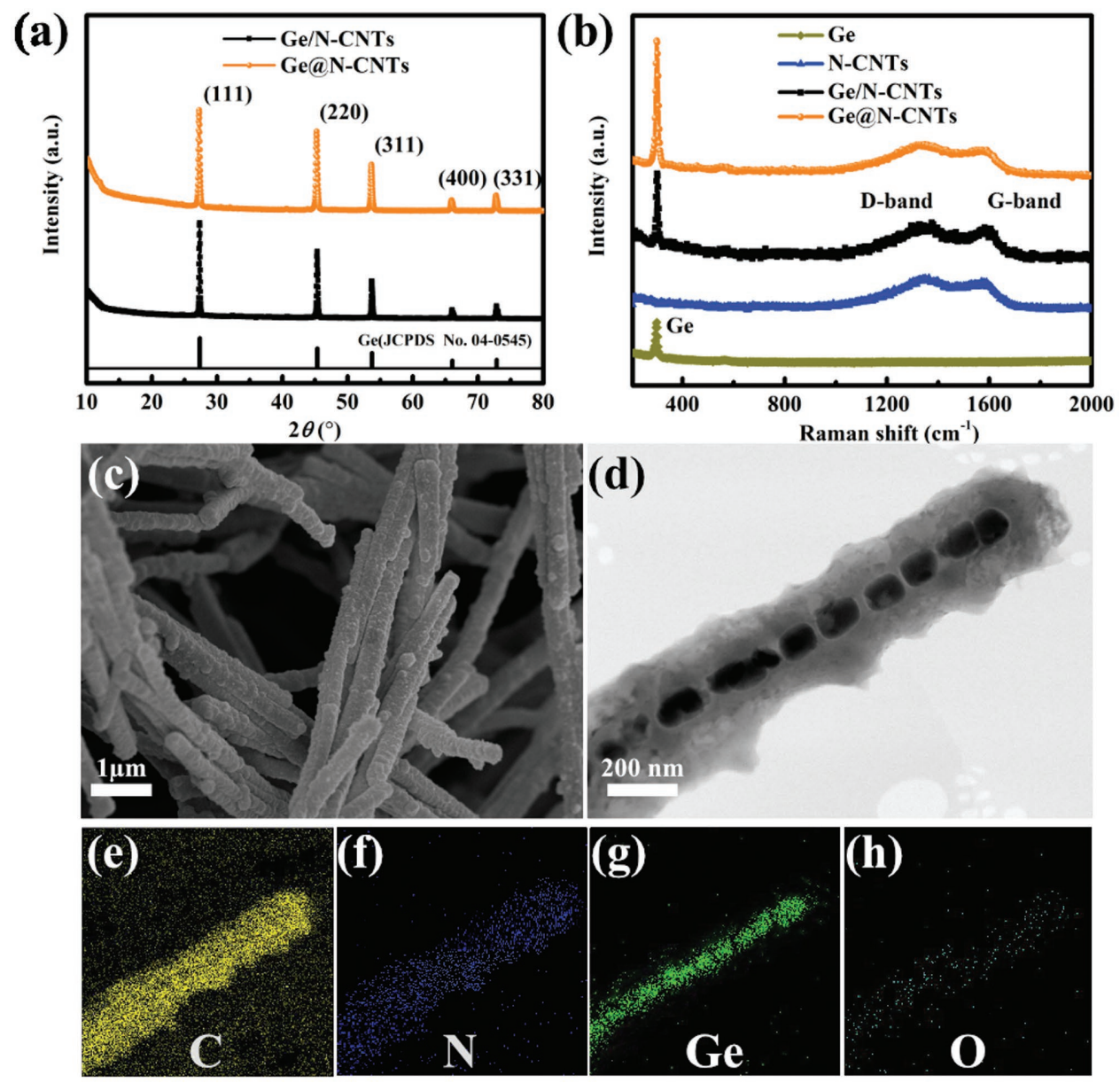

Figure 3. a) XRD patterns of Ge/N-CNTs and Ge@N-CNTs. b) Raman spectra of Ge, N-CNTs, Ge/N-CNTs, and Ge@N-CNTs. c) SEM and d) bright-field TEM images of Ge@N-CNTs. Elemental mapping images of e) C, f) N, g) Ge, and h) O of an individual Ge@N-CNTs nanotubes. 

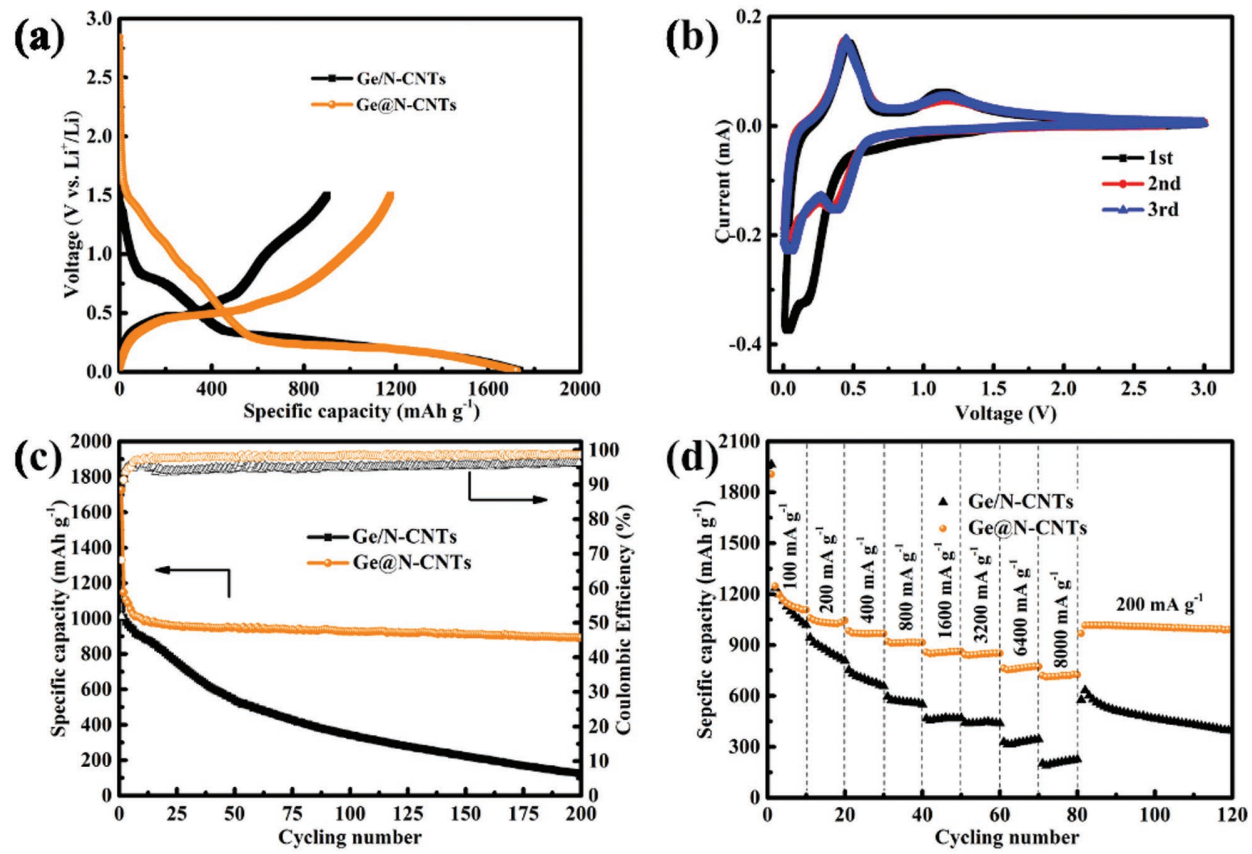

Figure 4. a) First cycle discharge/charge voltage curves of Ge/N-CNTs and Ge@N-CNTs composites cycled at a current density of 100 mA g ${ }^{-1}$ between 0.01 and $1.5 \mathrm{~V}$. b) Cyclic voltammetry profiles of Ge@N-CNTs at a scan rate of $0.1 \mathrm{mV} \mathrm{s}^{-1}$ for the first three cycles. c) Cycling stability of the Ge/N-CNTs and Ge@N-CNTs composites at a constant current density of $100 \mathrm{~mA} \mathrm{~g}^{-1}$ between 0.01 and $1.5 \mathrm{~V}$. d) Rate capabilities of Ge/N-CNTs and Ge@N-CNTs at different current densities.

Ge/N-CNTs could be assigned to the cubic Ge (JCPDS No. 04-0545) without any impurities, demonstrating that the $\mathrm{GeO}_{2}$ has been successfully reduced to $\mathrm{Ge}$ and that the $\mathrm{NaCl}$ has been fully removed. In addition, no obvious carbon diffraction peak can be detected because of the amorphous nature of the N-CNTs. The Raman spectra are shown in Figure 3b. For pure Ge, the peak centered at $300 \mathrm{~cm}^{-1}$ could be assigned to metallic Ge. As for the pure N-CNTs, peaks located at 1350 and $1580 \mathrm{~cm}^{-1}$ can be indexed to the $\mathrm{D}$ and $\mathrm{G}$ bands, respectively. Therefore, three peaks appeared at 300, 1350 , and $1580 \mathrm{~cm}^{-1}$ for the Ge@N-CNTs and Ge/N-CNTs, which can be attributed to metallic Ge, the D and $G$ bands of N-CNTs, respectively. It is worth mentioning that $\mathrm{FeCl}_{3}$ could be easily washed away after centrifugal treatment with ethanol and deionized water during the synthesis process of PPy nanotubes. In addition, there is no peak of residual Fe in the XRD patterns and Raman spectra, indicating that there is no Fe in the final product. As can be observed from the SEM image (Figure 3c), a large quantity of Ge@N-CNTs composite generally inherits the uniform tubular structure and diameter of the core-shell $\mathrm{GeO}_{2} / \mathrm{NaCl} @$ PPy nanotubes. The TEM image (Figure 3d and Figure S4, Supporting Information) further shows the details of the unique Ge@N-CNTs composite. The dark inner Ge cores are homogeneously encapsulated by the N-CNTs shells, and there are some void spaces not only between the individual Ge nanoparticles, but also between the Ge core and the N-CNTs shells. These uniformly distributed Ge nanoparticles could effectively use these void spaces to accommodate a large volume expansion during the cycling processes, thus preventing agglomeration of the electrode materials. It is notable that these void spaces are generated not only from the reduction treatment of $\mathrm{GeO}_{2}$ in $\mathrm{H}_{2}$ gas, but also from the removal of $\mathrm{NaCl}$ after centrifuge treatment. The elemental mapping results for $\mathrm{C}, \mathrm{N}$, and $\mathrm{Ge}$ further demonstrate that the Ge nanoparticles are uniformly distributed along the axial direction of the tube (Figure $3 \mathrm{e}-\mathrm{g}$ ). Moreover, the $\mathrm{N}$ doping in the CNTs could improve the electrical conductivity, which additionally contributes to the enhanced electrochemical performance. In addition, as shown in Figure 3h, the element mapping for oxygen is negligible, indicating that the $\mathrm{GeO}_{2}$ has been completely reduced to Ge during the annealing treatment. This maybe because the PPy nanotubes have open ends, which could allow the hydrogen gas flow into the cavities and reduce the $\mathrm{GeO}_{2}$. All of these data demonstrate the successful fabrication of this unique Ge@N-CNTs structure with Ge nanoparticles uniformly encapsulated in the N-CNTs.

To demonstrate the structural advantages of the Ge@N-CNTs, the electrochemical performances of both the Ge@N-CNTs and the Ge/N-CNTs composites were investigated. The specific capacity was calculated using both $\mathrm{N}$-CNTs and Ge composite. Figure 4 a presents the first cycle discharge/charge curves of the Ge@N-CNTs and Ge/N-CNTs composites at a current density of $100 \mathrm{~mA} \mathrm{~g}^{-1}$ in the potential range of $0.01-1.5 \mathrm{~V}$ versus $\mathrm{Li} / \mathrm{Li}^{+}$. The $\mathrm{Ge} / \mathrm{N}-\mathrm{CNT}$ s exhibit initial discharge and charge capacities of 1737 and $900 \mathrm{mAh} \mathrm{g}^{-1}$, respectively, with a Coulombic efficiency of $52 \%$. On the other hand, the Ge@N-CNTs composite shows a higher initial Coulombic efficiency of $68 \%$, and its initial discharge and charge capacities are 1725 and $1176 \mathrm{mAh} \mathrm{g}^{-1}$, respectively. The lithium storage mechanism of the Ge@N-CNTs was investigated by using cyclic voltammetry (CV), as shown in Figure $4 \mathrm{~b}$. There is a small reduction peak located at $0.16 \mathrm{~V}$ in the first cycle, which could be attributed to the formation 
of Li-Ge phase. ${ }^{[43-46]}$ This peak disappears, however, after the first cycle, and new peak appears at $0.36 \mathrm{~V}$, suggesting the phase transition from lithiated $\mathrm{Li}_{x} \mathrm{Ge}$ to amorphous Ge. ${ }^{[47]}$ In the case of Ge/N-CNTs, there is an obvious reduction peak centered at $1.3 \mathrm{~V}$ in the first cycle, which could be assigned to the formation of a solid electrolyte interphase (SEI) film between the Ge nanoparticles and the electrolyte (Figure S5, Supporting Information). Figure $4 \mathrm{c}$ presents the cycling stability of the Ge@N-CNTs and Ge/N-CNTs composites at a constant current density of $100 \mathrm{~mA} \mathrm{~g}^{-1}$ in the potential range of 0.01-1.5 V. Clearly, the Ge@N-CNTs presents an enhanced cycling stability and higher reversible capacity compared to the Ge/N-CNTs. Specifically, the Ge@N-CNTs composite still delivers a high discharge capacity of $892 \mathrm{mAh} \mathrm{g}^{-1}$ beyond 200 cycles. Moreover, it demonstrates better Coulombic efficiency compared to the Ge/N-CNTs, showing the better reversibility of the Ge@N-CNTs (Figure 4c and Figure S6, Supporting Information). The rapid capacity fading is due to the huge volume changes during the cycling process, which would not only lead to the pulverization and loss of active material, but also the continuous regeneration of the SEI film. As for the Ge@N-CNTs, the enhanced cycling stability demonstrates the advantages of the unique 1D structure, which could preserve the stable SEI films by accommodating the large volume expansion. The rate capabilities of the Ge@N-CNTs and Ge/N-CNTs composites were also investigated at various current densities.The Ge@N-CNTs shows considerably improved rate performance when compared with the Ge/N-CNTs at all high current densities (Figure 4d). The specific capacities of the Ge@N-CNTs composite at the current densities of 3200 and $6400 \mathrm{~mA} \mathrm{~g}^{-1}$ were still 850 and $770 \mathrm{mAh} \mathrm{g}{ }^{-1}$, respectively. The Ge@N-CNTs still deliver a reversible capacity of $725 \mathrm{mAh} \mathrm{g}^{-1}$, even at the high current rate of $8000 \mathrm{~mA} \mathrm{~g}^{-1}$, whereas the Ge/N-CNTs only exhibit an average capacity of $220 \mathrm{mAh} \mathrm{g}^{-1}$ at the current rate of $8000 \mathrm{~mA} \mathrm{~g}^{-1}$. In addition, the Ge@N-CNTs composite shows very good cycling stability at high current density. As shown in Figure S7 (Supporting Information), Ge@N-CNTs still deliver a reversible capacity of $715 \mathrm{mAh} \mathrm{g}^{-1}$ over 180 cycles at the current density of $8000 \mathrm{~mA} \mathrm{~g}^{-1}$. The enhanced electrochemical performance of the Ge@N-CNTs composite could be attributed to the unique Ge nanoparticles encapsulated in the N-CNTs shells. Specifically, the Ge nanoparticles could facilitate $\mathrm{Li}^{+}$ion transport through reducing the diffusion distance. Furthermore, the void spaces and the N-CNTs could effectively suppress the huge volume expansion during cycling processes. In addition, the interconnected network of the N-CNTs could afford good electrical conductivity of the electrode and prevent electrical isolation after prolonged cycling. Therefore, when investigated as anode material, the Ge@N-CNTs composite manifests high specific capacity, enhanced cycling stability, and excellent rate capacity. The structural stability of the Ge@N-CNTs and Ge/N-CNTs was also investigated with ex situ TEM after alloying and dealloying. As can be seen from the TEM image (Figure S6a, Supporting Information), the 1D structure of the Ge@N-CNTs is well preserved after full alloying. $\mathrm{Li}_{x} \mathrm{Ge}$ completely fills the inner hollow cavity, and the diameter of the nanotube increases from $300 \mathrm{~nm}$ to about $500 \mathrm{~nm}$, indicating that the void spaces and N-CNTs could effectively accommodate the volume expansion. Moreover, the structure recovered well after the dealloying process, and the Ge nanoparticles became porous after the extraction of $\mathrm{Li}$ during the recharge process (Figure S6b, Supporting Information), which is consistent with previous reports. ${ }^{[4,49]}$ For the Ge/N-CNTs composite, on the other hand, the bare Ge nanoparticles aggregated together after full lithiation, with the diameter of the N-CNTs slightly increased from 300 to $330 \mathrm{~nm}$ (Figure S6c, Supporting Information). Furthermore, after dealloying, the size of the Ge particles became significantly smaller after the extraction of Li (Figure S6d, Supporting Information), which would lead to the continuous regeneration of SEI film in the following cycles. As shown in Figure S9 (Supporting Information), even after 200 cycles, the unique 1D structure of the Ge@N-CNTs could be well preserved. These results imply enhanced structural stability of the unique 1D structure, with Ge nanoparticles uniformly distributed in the N-CNTs during cycling, which results in the enhanced electrochemical performance of the Ge@N-CNTs.

In conclusion, we have presented a novel strategy to fabricate Ge@N-CNTs composite with Ge nanoparticles uniformly encapsulated in N-CNTs shells by using capillary action. Through adding PVP, the high-surface-tension $\mathrm{GeO}_{2} /$ $\mathrm{NaCl}$ solution could be induced into the inner hollow cavities of the PPy nanotubes to form a core-shell $\mathrm{GeO}_{2} / \mathrm{NaCl} @ P P y$ nanotubes composite. After further reducing $\mathrm{GeO}_{2}$ to $\mathrm{Ge}$ and removing $\mathrm{NaCl}$, the unique Ge@N-CNTs composite with Ge nanoparticles uniformly distributed in the N-CNTs could be obtained.The Ge@N-CNTs demonstrate enhanced cycling stability and excellent rate capability in comparison with $\mathrm{Ge} / \mathrm{N}-\mathrm{CNTs}$, which could be attributed to the efficiently utilization of the void spaces provided by the uniformly distributed Ge nanoparticles. Moreover, the present strategy could be a general method to fabricate other 1D structured materials with enhanced electrochemical performance.

\section{Experimental Section}

Synthesis of PPy Nanotubes: PPy nanotubes were synthesized using a previously reported method. ${ }^{[50]}$ In a typical synthesis process, methyl orange $\left(0.25 \times 10^{-3} \mathrm{M}\right)$ was added in deionized water $(100 \mathrm{~mL})$, and then $\mathrm{FeCl}_{3}(1.35 \mathrm{~g})$ was dissolved to the solution. Then, the pyrrole monomer $\left(5 \times 10^{-3} \mathrm{M}\right)$ was dissolved into the mixture dropwise. The solution was stirred for $24 \mathrm{~h}$ at room temperature. The thus-formed PPy nanotubes were washed with ethanol and deionized water several times. Then, PPy nanotubes with an inner cavity about $100 \mathrm{~nm}$ in diameter were obtained.

Synthesis of Ge@N-CNTs: PPy nanotubes $(50 \mathrm{mg})$ were first added into deionized water $(50 \mathrm{~mL})$, followed by ultrasonication for $1.5 \mathrm{~h}$ to form a suspension. Meanwhile, $\mathrm{GeO}_{2}(144 \mathrm{mg})$ were dissolved in $\mathrm{NaOH}$ solution $(100 \mathrm{~mL})$. After stirring for $1.5 \mathrm{~h}$, the two solutions were mixed together, and then vigorously stirred for $20 \mathrm{~min}$. The $\mathrm{pH}$ of the solution was slowly adjusted to 7 with dilute $\mathrm{HCl}$. Then, PVP (MW = 10 000) (8 mg) was added into the solution. After drying out, the sample was annealed to $650{ }^{\circ} \mathrm{C}$ in a tube furnace at a rate of $5^{\circ} \mathrm{C} \mathrm{min}-1$ in argon atmosphere. When the temperature reached $650{ }^{\circ} \mathrm{C}$, the argon gas was replaced by $\mathrm{H}_{2}$ gas, 
and the reaction proceeds for $4 \mathrm{~h}$. Finally, the sample was left to cool down to room temperature and then washed with ethanol and deionized water several times. To synthesize Ge/N-CNTs, the same method is used, but without adding PVP.

Materials Characterization: The crystalline structures of the samples were analyzed by powder XRD (GBC MMA) using $\mathrm{Cu} \mathrm{K} \alpha$ radiation. Raman spectra were carried out by using JOBIN YVON HR800 Confocal system. Thermogravimetric analysis (TGA) tests were collected with a TGA instrument (Mettler-Toledo, Switzerland) from room temperature to $800{ }^{\circ} \mathrm{C}$ at a rate of $10{ }^{\circ} \mathrm{C} \mathrm{min}-1$ in air. The morphologies of the materials were investigated by scanning electron microscopy (JSM-7500FA, JEOL) and transmission electron microscopy (JEM-ARM200F, JEOL).

Electrochemical Measurement: 2032-type coin-cells were used to test the electrochemical performance. The electrodes were prepared by mixing the active material ( 80 wt\%), Super $\mathrm{P}$ (10 wt\%), and sodium carboxymethyl cellulose (10 wt\%) in deionized water. The slurry was then cast on Cu foil and dried at $80^{\circ} \mathrm{C}$ for $12 \mathrm{~h}$ in a vacuum oven. The working electrodes were prepared by punching the $\mathrm{Cu}$ film into discs $0.97 \mathrm{~cm}$ in diameter, and the loading mass of the active material for all the electrodes was at least $1.1 \mathrm{mg} \mathrm{cm}^{-2}$. The coin-cells were assembled in an argon-filled glove box (Mbraun, Germany) with the prepared active material on Cu foil as working electrode, lithium foil as counter electrode, microporous polyethylene (Celgard 2400) as the separator, and $1 \mathrm{M} \mathrm{LiPF}_{6}$ in a mixture of ethylene carbonate (EC), diethylcarbonate (DEC), and dimethyl carbonate (DMC) (3:4:4 by volume) as electrolyte. The assembly process was carried out in an argon-filled glove box with the oxygen and humidity levels under $0.1 \mathrm{ppm}$ (Mbraun, Germany). The galvanostatically discharge and charge processes were carried out on a Land battery tester between 0.01 and $1.5 \mathrm{~V}$ versus $\mathrm{Li}^{+} / \mathrm{Li}$. Cyclic voltammetry was conducted with a Biologic VMP-3 electrochemical workstation.

\section{Supporting Information}

Supporting Information is available from the Wiley Online Library or from the author.

\section{Acknowledgements}

This research was financially supported by the Baosteel-Australia Joint Research and Development Centre (BA14006). The authors thank Dr. Tania Silver for critical reading of the manuscript, and acknowledge the use of the facilities in the University of Wollongong Electron Microscopy Center.

\section{Conflict of Interest}

The authors declare no conflict of interest.

[1] B. Dunn, H. Kamath, J. M. Tarascon, Science 2011, 334, 928

[2] S. Xin, Z. Chang, X. Zhang, Y. Guo, Natl. Sci. Rev. 2016, 4, 1759.
[3] J. R. Dahn, T. Zheng, Y. Liu, J. S. Xue, Science 1995, 270, 590.

[4] W. Xiao, J. Zhou, L. Yu, D. Wang, X. W. Lou, Angew. Chem. Int. Ed. 2016, 55, 7427.

[5] T. Kennedy, M. Brandon, K. M. Ryan, Adv. Mater. 2016, 28, 5696.

[6] S. Wu, C. Han, J. locozzia, M. Lu, R. Ge, R. Xu, Z. Lin, Angew. Chem. Int. Ed. 2016, 55, 7898.

[7] K. H. Seng, M. H. Park, Z. P. Guo, H. K. Liu, J. Cho, Nano Lett. 2013 , $13,1230$.

[8] K. H. Seng, M. H. Park, Z. P. Guo, H. K. Liu, J. Cho, Angew. Chem. Int. Ed. 2012, 51, 5657.

[9] G. Cui, L. Gu, N. Kaskhedikar, P. A. van Aken, J. Maier, Electrochim. Acta 2010, 55, 985.

[10] D. J. Xue, S. Xin, Y. Yan, K. C. Jiang, Y. X. Yin, Y. G. Guo, L. J. Wan, J. Am. Chem. Soc. 2012, 134, 2512.

[11] X. Liu, J. Hao, X. Liu, C. Chi, N. Li, F. Endres, Y. Zhang, Y. Li, J. Zhao, Chem. Commun. 2015, 51, 2064.

[12] W. Li, M. Li, Z. Yang, J. Xu, X. Zhong, J. Wang, L. Zeng, X. Liu, Y. Jiang, X. Wei, L. Gu, Y. Yu, Small 2015, 11, 2762.

[13] F. W. Yuan, H. J. Yang, H. Y. Tuan, ACS Nano 2012, 6, 9932.

[14] M. H. Park, Y. Cho, K. Kim, J. Kim, M. Liu, J. Cho, Angew. Chem. Int. Ed. 2011, 50, 9647.

[15] J. Liu, K. Song, C. Zhu, C. C. Chen, P. A. van Aken, J. Maier, Y. Yu, ACS Nano 2014, 8, 7051.

[16] Z. Chen, Y. Yan, S. Xin, W. Li, J. Qu, Y. G. Guo, W. G. Song, J. Mater. Chem. A 2013, 1, 11404.

[17] S. Xin, L. Yu, Y. You, H. P. Cong, Y. X. Yin, X. L. Du, Y. G. Guo, S. H. Yu, Y. Cui, J. B. Goodenough, Nano Lett. 2016, 16, 4560.

[18] Y. Sun, S. Jin, G. Yang, J. Wang, C. Wang, ACS Nano 2015, 9 , 3479.

[19] W. J. Yu, C. Liu, P. X. Hou, L. Zhang, X. Y. Shan, F. Li, H. M. Cheng, ACS Nano 2015, 9, 5063.

[20] J. Q. Hu, X. M. Meng, Y. Jiang, C. S. Lee, S. T. Lee, Adv. Mater. 2003, 15, 70 .

[21] A. Pandurangan, C. Morin, D. Qian, R. Andrews, M. Crocker, Carbon 2009, 47, 1708.

[22] G. A. Domrachev, A. M. Ob’edkov, B. S. Kaverin, A. A. Zaitsev, S. N. Titova, A. I. Kirillov, A. S. Strahkov, S. Y. Ketkov, E. G. Domracheva, K. B. Zhogova, Chem. Vap. Deposition 2006, $12,357$.

[23] Y. Yu, L. Gu, C. Wang, A. Dhanabalan, P. A. van Aken, J. Maier, Angew. Chem. Int. Ed. 2009, 48, 6485.

[24] X. Zhou, L. Yu, X. Y. Yu, X. W. D. Lou, Adv. Energy Mater. 2016, 6, 1601177.

[25] S. Xin, Y. G. Guo, L. J. Wan, Acc. Chem. Res. 2012, 45, 1759.

[26] B. Jiang, Y. He, B. Li, S. Zhao, S. Wang, Y. He, Z. Lin, Angew. Chem. Int. Ed. 2017, 56, 1869.

[27] B. Jiang, C. Han, B. Li, Y. He, Z. Lin, ACS Nano 2016, 10, 2728.

[28] S. Zhao, Z. Wang, Y. He, B. Jiang, Y. Harn, X. Liu, F. Yu, F. Feng, Q. Shen, Z. Lin, ACS Energy Lett. 2017, 2, 111

[29] K. Huo, L. Wang, C. Peng, X. Peng, Y. Li, Q. Li, Z. Jin, P. K. Chu, J. Mater. Chem. A 2016, 4, 7585.

[30] C. Wu, Y. Jiang, P. Kopold, P. A. van Aken, J. Maier, Y. Yu, Adv. Mater. 2016, 28, 7276.

[31] C. Wu, P. Kopold, P. A. van Aken, J. Maier, Y. Yu, Adv. Mater. 2017, 29, 1604015

[32] W. Li, Z. Yang, J. Cheng, X. Zhong, L. Gu, Y. Yu, Nanoscale 2014, 6, 4532.

[33] S. C. Tsang, Y. K. Chen, P. J. F. Harris, M. L. H. Green, Nature 1994, $372,159$.

[34] R. Kreizman, S. Y. Hong, J. Sloan, R. Popovitz-Biro, A. Albu-Yaron, G. Tobias, B. Ballesteros, B. G. Davis, M. L. Green, R. Tenne, Angew. Chem. Int. Ed. 2009, 48, 1230.

[35] G. Zheng, Q. Zhang, J. J. Cha, Y. Yang, W. Li, Z. W. Seh, Y. Cui, Nano Lett. 2013, 13, 1265 .

[36] M. Haft, M. Grönke, M. Gellesch, S. Wurmehl, B. Büchner, M. Mertig, S. Hampel, Carbon 2016, 101, 352.

[37] Y. Zou, Y. Wang, ACS Nano 2011, 5, 8108. 
[38] T. P. Kumar, R. Ramesh, Y. Y. Lin, G. T.-K. Fey, Electrochem. Commun. 2004, 6, 520.

[39] D. Ugarte, A. Chatelain, W. A. de Heer, Science 1996, 274, 1897.

[40] E. Dujardin, T. W. Ebbesen, H. Hiura, K. Tanigaki, Science 1994, 265, 1850.

[41] A. Mizev, A. Trofimenko, D. Schwabe, A. Viviani, Eur. Phys. J.: Spec. Top. 2013, 219, 89.

[42] E. Kim, Y. Xia, G. M. Whitesides, J. Am. Chem. Soc. 1996, 118, 5722.

[43] Y. Xiao, M. Cao, Chem. Asian J. 2014, 9, 2859.

[44] C. Zhong, J. Z. Wang, X. W. Gao, D. Wexler, H. K. Liu, J. Mater. Chem. A 2013, 1, 10798.

[45] S. Fang, L. F. Shen, H. Zheng, X. G. Zhang, J. Mater. Chem. A 2015, 3, 1498 .
[46] Y. Xu, X. S. Zhu, X. S. Zhou, X. Liu, Y. X. Liu, Z. H. Dai, J. C. Bao, J. Phys. Chem. C 2014, 118, 28502.

[47] C. Yan, W. Xi, W. Si, J. Deng, O. G. Schmidt, Adv. Mater. 2013, 25, 539.

[48] T. Kennedy, E. Mullane, H. Geaney, M. Osiak, C. O'Dwyer, K. M. Ryan, Nano Lett. 2014, 14, 716.

[49] X. H. Liu, S. Huang, S. T. Picraux, J. Li, T. Zhu, J. Y. Huang, Nano Lett. 2011, 11, 3991.

[50] M. Li, W. Li, J. Liu, J. Yao, J. Mater. Sci.: Mater. Electron. 2013, 24 , 906.

Received: March 21, 2017

Revised: April 7, 2017

Published online: May 30, 2017 